\title{
POPULATION STUDY ARTICLE Metabolomic profiles and development of metabolic risk during the pubertal transition: a prospective study in the ELEMENT Project
}

\author{
Wei Perng ${ }^{1}$, Lu Tang ${ }^{2}$, Peter X. K. Song ${ }^{2}$, Martha Maria Tellez-Rojo ${ }^{3}$, Alejandra Cantoral ${ }^{4}$ and Karen E. Peterson ${ }^{1}$
}

\begin{abstract}
OBJECTIVES: (1) Examine associations of a branched-chain amino acid (BCAA) metabolite pattern with metabolic risk across adolescence; (2) use Least Absolute Shrinkage and Selection Operator (LASSO) to identify novel metabolites of metabolic risk. METHODS: We used linear regression to examine associations of a BCAA score with change $(\Delta)$ in metabolic biomarkers over 5-year follow-up in 179 adolescents 8-14 years at baseline. Next, we applied LASSO, a regularized regression technique well suited for reduction of high-dimensional data, to identify metabolite predictors of $\triangle$ biomarkers.

RESULTS: In boys, the BCAA score corresponded with decreasing C-peptide, C-peptide-based insulin resistance (CP-IR), total cholesterol (TC), and low-density-lipoprotein cholesterol (LDL). In pubertal girls, the BCAA pattern corresponded with increasing C-peptide and leptin. LASSO identified asparagine as a predictor of decreasing C-peptide $(\beta=-0.33)$ and CP-IR $(\beta=-0.012)$, and acetyl-carnitine $(\beta=2.098)$, 4-hydroxyproline $(\beta=-0.050)$, ornithine $(\beta=-0.353)$, and a-aminoisobutyric acid $(\beta=-0.793)$ as determinants of TC in boys. In girls, histidine was a negative determinant of TC $(\beta=-0.033)$.

CONCLUSIONS: The BCAA pattern was associated with $\triangle$ glycemia and $\Delta$ lipids in a sex-specific manner. LASSO identified asparagine, which influences growth hormone secretion, as a determinant of decreasing C-peptide and CP-IR in boys, and metabolites on lipid metabolism pathways as determinants of decreasing cholesterol in both sexes.

Pediatric Research (2019) 85:262-268; https://doi.org/10.1038/s41390-018-0195-5
\end{abstract}

\section{INTRODUCTION}

Profiling of circulating metabolites, known as metabolomics, shows promise as one route to identifying specific targets for primary prevention. In the realm of obesity-related disease, studies in adults have unveiled distinct differences in plasma metabolite composition of obese vs. lean persons, ${ }^{1}$ some of which precede development of insulin resistance and type 2 diabetes by over a decade, independently of weight status. ${ }^{2}$ These findings suggest that metabolite patterns have higher discriminative capacity than weight status or traditional metabolic biomarkers to identify persons at risk of type 2 diabetes earlier on the disease continuum, and that some metabolite patterns may signal risk even among non-overweight/obese individuals.

Less is known of these relationships earlier in the life course, when health trajectories are more malleable. Despite a flurry of analyses exploring cross-sectional associations of circulating metabolites with conventional biomarkers of glycemia in children and adolescents, ${ }^{3-6}$ only two studies have interrogated this relationship prospectively: one investigation followed 17 adolescents in Boston over the course of 18 months, ${ }^{6}$ and another was a 2-year study of 102 Korean boys. ${ }^{7}$ The scant literature in youth is problematic for both biological and methodological reasons. Biologically, understanding determinants and etiology of worsening metabolic health during early life is essential for effective prevention. Methodologically, replication of existing findings is critical, especially in light of inconsistencies in current literature in the area of metabolomics and metabolic health, ${ }^{8}$ and the potential for false-positive associations in analyses of high-dimensional 'omics data.

Here, we address the above-mentioned gaps via a two-pronged approach comprised of a hypothesis-driven and a data-driven analytical strategy. First, we investigated associations of a branched-chain amino acid (BCAA) metabolite pattern that has been previously associated with metabolic risk in youth in crosssectional analyses, ${ }^{5-7,9}$ with change in glycemia, adipokines, lipid profile, and blood pressure during 5 years of follow-up in a cohort of Mexican children aged 8-14 years at baseline. Second, based on results from the first step (i.e., significant relationships between BCAA and the metabolic biomarkers), we applied Least Absolute Shrinkage and Selection Operator (LASSO) regression on the metabolite data set to identify the strongest metabolite determinants of change in conventional biomarkers of interest during follow-up. This procedure efficiently handles high-dimensional correlated predictors, and is useful for validation of hypothesisdriven results. Additionally, because we used results from the hypothesis-driven analysis to inform outcomes of interest for LASSO, this approach reduces the number of comparisons being made.

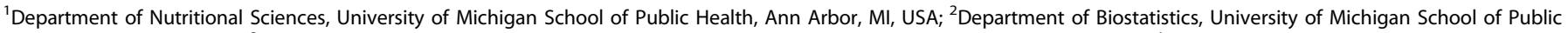

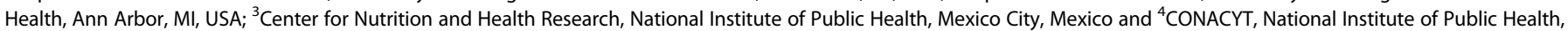
Center for Research on Nutrition and Health, Mexico City, Mexico

Correspondence: Wei Perng (wei.perng@gmail.com)

Received: 20 March 2018 Revised: 22 August 2018 Accepted: 10 September 2018

Published online: 3 October 2018 


\section{MATERIALS AND METHODS}

Study population

This study included participants of the Early Life Exposure in Mexico to Environmental Toxicants (ELEMENT) Project, a cohort study of pregnant women and their offspring in Mexico City, Mexico. Details on recruitment and eligibility have been previously published. ${ }^{10}$ In brief, we recruited women from public maternity hospitals in Mexico City between 1997 and 2004 and followed these women and their children up to 5 years post partum. In 2010, we re-contacted a subset of the offspring, who were then 8-14 years of age, based on availability of archived prenatal biospecimens $(n=250)$ to participate in follow-up studies. At inperson research visits that took place in 2010 (henceforth, referred to as the "baseline visit"), the children provided an 8-h fasting blood sample and participated in anthropometric assessment. Approximately 5 years later, when the children were 13-19 years of age, we carried out another research visit to obtain fasting blood and measure anthropometry ("follow-up visit"). Of the 207 children who attended both visits, the present analysis includes 179 participants who had adequate fasting serum volume from the baseline visit for the targeted metabolomics assays, and data on at least one of the conventional metabolic biomarkers of interest at both baseline and follow-up. The institutional review boards of the Mexico National Institute of Public Health and the University of Michigan approved research protocols. The study was carried out in accordance with the approved guidelines and regulations, including appropriate informed consent (maternal informed consent and child assent).

Targeted metabolomics profiling

The Michigan Regional Comprehensive Metabolomics Resource Core $\left(\mathrm{MRC}^{2}\right.$ ) qucantified absolute concentrations of 22 amino acids ${ }^{11}$ and 27 acylcarnitine species ${ }^{12}$ from fasting serum collected at baseline. Coefficients of variation for both chemical classes were $<10 \%$ within a day and $<15 \%$ between days. Details on laboratory methods are provided in the Supplemental Material.

\section{Conventional metabolic biomarkers}

The outcomes of interest were change in conventional biomarkers of metabolic risk, calculated as the difference in the biomarker level at follow-up minus biomarker level at baseline. For this analysis, we considered markers of glycemia, as indicated by fasting glucose, C-peptide as a surrogate for fasting insulin, ${ }^{13}$ and C-peptide-based insulin resistance (CP-IR); leptin, an adiposetissue-derived adipokine involved in glycemic regulation; lipid profile, according to total cholesterol, triglycerides high-density lipoprotein (HDL), and low-density lipoprotein (LDL); systolic blood pressure (SBP) and diastolic blood pressure (DBP); and a metabolic syndrome risk $z$-score (MetRisk $z$-score).

Fasting glucose, C-peptide, and leptin. Using fasting blood, we measured serum glucose enzymatically. Serum C-peptide was quantified using an automated chemiluminescence immunoassay (Immulite 1000, Siemens Medical Solutions). These biomarkers provide a measure of glycemic control as fasting glucose is an indicator of glucose metabolism and a diabetes screening tool, and $C$-peptide is a marker of insulin secretory function that is secreted in quantities equal to insulin from pancreatic $\beta$ cells. ${ }^{13}$ We measured leptin from serum using a radioimmunoassay (Millipore).

Lipid profile. We measured total cholesterol, triglycerides, and HDL cholesterol (HDL-C) in peripubertal fasting serum samples $(\mathrm{mg} / \mathrm{dL})$ using a biochemical analyzer (Cobas Mira Plus, Roche Diagnostics), and calculated LDL cholesterol (LDL-C) as: total cholesterol - HDL-C - (triglycerides/5).

Blood pressure. Research staff measured SBP and DBP in duplicate to the nearest $\mathrm{mmHg}$ in the seated position using
BpTRU monitors (Coquitlam, BC, Canada), and used the average of the repeated measures for the analysis.

MetRisk z-score. For the MetRisk z-score, we calculated the sum of five age-specific and sex-specific internal $z$-scores for waist circumference, fasting glucose, fasting C-peptide, triglycerides/ $\mathrm{HDL}-\mathrm{C}$, and SBP + DBP)/2. We have previously published this score in the ELEMENT cohort with respect to diet, ${ }^{14,15}$ metabolomics, ${ }^{16}$ and early growth. ${ }^{17}$ This score is a modification (i.e., use of Cpeptide rather than insulin) of one proposed by Ekelund et al., ${ }^{18}$ which was then validated by Viitasalo et al. ${ }^{19}$ in a study that examined correlations among the metabolic biomarkers in children and adults, and established its association with incident type 2 diabetes and cardiovascular disease across the life course.

\section{Covariates}

Upon enrollment, mothers reported on age, reproductive history, smoking history, and sociodemographic characteristics. A pediatrician trained in standardized methods assessed each child at the baseline and follow-up visits to determine Tanner stage on a scale of 1 (no development) to 5 (full development) for testicle (boys), breast (girls), and pubic hair development (both). For the analysis, we dichotomized pubertal status as prepubertal vs. pubertal: boys were considered pubertal if they received an assessment of Tanner stage $>1$ for genital or pubic hair development, and girls were considered pubertal if they received an assessment of Tanner stage $>1$ for breast or pubic hair development.

Data analysis

Hypothesis-driven approach. The goal of this approach was to examine associations of a previously characterized BCAA metabolite pattern with change in several metabolic biomarkers during the follow-up period. To create the BCAA pattern, we selected the following compounds: the BCAAs valine, leucine, and isoleucine; the large neutral amino acids phenylalanine, tyrosine, and tryptophan, which share the same cellular transport mechanism (LAT1) as the BCAAs; ${ }^{20}$ alanine, an aliphatic amino acid linked to type 2 diabetes risk via its role in the glucose-alanine cycle; ${ }^{21}$ and propionyl-carnitine (C3) and valeryl-carnitine (C5:0), which are downstream catabolites of BCAA metabolism. We derived the BCAA factor score by entering these compounds into an unsupervised principal components analysis. Judging from the Scree plot (Supplemental Figure S1), we retained the first factor for its clear dominance in accounting for the total variability. The factor retained represents a continuous, normally distributed weighted score of the compounds that compose the BCAA metabolite pattern based on their natural intercorrelations. The higher score for the BCAA pattern, the greater the degree to which an individual's circulating metabolite profile resembles this pattern.

Next, we examined associations of the BCAA factor score with change in the metabolic biomarkers during follow-up, while also adjusting for the child's baseline age, pubertal status, and biomarker level; and age at follow-up (Model 1). We then further adjusted for body mass index (BMI) $z$-score at baseline (Model 2) since weight status independently may impact subsequent metabolic health. Due to evidence of effect modification by sex for several biomarkers, and in light of known differences in physiology between boys and girls during adolescence, we ran all models separately by sex. Because we found statistical evidence of an interaction between the BCAA pattern and pubertal status in girls, we also ran models separately for pre-pubertal and pubertal females.

In addition to examining associations with the BCAA factor score, we evaluated individual metabolites within the BCAA pattern using Model 2. We parameterized the metabolites as a $z-$ score centered at the median and scaled by $2 / 3$ of the mean 
Table 1. Mean \pm SD factor scores, and change $(\Delta)$ in body composition and metabolic biomarkers between 8-13 years of age (baseline) and 13-18 years of age (follow-up) among 179 ELEMENT participants

\begin{tabular}{|c|c|c|c|c|}
\hline & \multicolumn{2}{|l|}{ Boys $(n=85)$} & \multicolumn{2}{|l|}{ Girls $(n=94)$} \\
\hline BCAA pattern factor score & $-0.08 \pm 0.86$ & & $0.13 \pm 1.14$ & \\
\hline Age (years) & $10.2 \pm 1.6$ & $13.6 \pm 1.8$ & $10.0 \pm 1.6$ & $13.3 \pm 1.7$ \\
\hline Waist circumference $(\mathrm{cm})$ & $69.7 \pm 9.8$ & $77.1 \pm 11.0$ & $70.2 \pm 10.1$ & $79.4 \pm 10.4$ \\
\hline C-peptide (ng/mL) & $1.51 \pm 1.09$ & $0.40 \pm 0.24$ & $1.81 \pm 1.38$ & $77.1 \pm 6.6$ \\
\hline Leptin $(\mathrm{ng} / \mathrm{mL})$ & $8.7 \pm 6.8$ & $15.2 \pm 13.1$ & $13.3 \pm 9.3$ & $25.4 \pm 17.9$ \\
\hline Total cholesterol (mg/dL) & $153.7 \pm 27.0$ & $146.6 \pm 23.2$ & $163.0 \pm 26.1$ & $159.2 \pm 23.8$ \\
\hline $\mathrm{HDL}(\mathrm{mg} / \mathrm{dL})$ & $60.0 \pm 11.8$ & $42.9 \pm 8.0$ & $57.8 \pm 12.6$ & $44.2 \pm 9.7$ \\
\hline LDL (mg/dL) & $80.3 \pm 23.7$ & $85.6 \pm 19.7$ & $85.3 \pm 21.0$ & $92.7 \pm 19.1$ \\
\hline Triglycerides (mg/dL) & $78.9 \pm 38.5$ & $90.3 \pm 44.7$ & $99.0 \pm 48.7$ & $111.4 \pm 64.9$ \\
\hline
\end{tabular}

${ }^{a}$ Calculated as the average of five internally standardized sex-specific $z$-scores for waist circumference, fasting glucose, fasting C-peptide, ratio of triglycerides to $\mathrm{HDL}$, and the average of SBP + DBP

absolute deviation from the median. This enabled us to obtain a more granular understanding of specific compounds/pathways that may be driving the relationships of interest.

Data-driven approach. We applied LASSO regression ${ }^{22}$ on all 22 amino acids and 27 acylcarnitines to identify metabolites that are predictive of change in biomarkers that were "predicted" by the BCAA pattern in the first part of the analysis. In other words, if the BCAA pattern was associated with change in a biomarker in conventional multivariable linear regression, then we used LASSO to identify metabolites predictive of that biomarker.

The mechanics of LASSO have been previously described. ${ }^{22}$ In brief, LASSO is a regularized regression technique that detects the strongest signals (in this case, metabolites) from a highdimensional and correlated set of predictors. We employed this method for its resistance to model over-fitting and detection of false-positive associations, which are issues when dealing with high-dimensional data (when $P » n$ ). The key feature of LASSO is the imposition of a constraint on model coefficients $(\beta$, representing the relationship between a given set of metabolite and change in the biomarker of interest, while conditioning on all other metabolites in the data set and adjusting for key covariates) so that week or null estimates are "shrunk" to zero and removed from the predictive model. This shrinkage procedure for variable selection is particularly useful for highdimensional 'omics data, as it reduces the possibility of falsepositive associations that may arise when metabolites exhibiting small and likely biologically irrelevant associations with the response variable are retained on the basis of statistical significance. To identify the $\beta$ below which the coefficient for a given metabolite is set to 0 , we used 10 -fold cross-validation to select the threshold that minimizes model prediction error. ${ }^{23}$ Of note, it is currently not possible to obtain $P$ values or confidence intervals from LASSO using available statistical software as the shrinkage process adds uncertainty in model selection, which is difficult to precisely quantify.

With the exception of the LASSO regression and crossvalidation, which were carried out using the glmnet package (Version 2.0-13) in R, we performed all analyses using Statistical Analyses System 9.3 software (SAS Institute Inc., Cary, NC, USA).

\section{RESULTS}

Median age of the participants at the baseline visit was 9.8 years (range: $8.1-13.8$ years); $47.8 \%(n=99)$ were boys. Mean \pm SD of the BCAA factor score, age, and change in the metabolic biomarkers at the mid-childhood and early teen visits are presented in Table 1.

Table 2 shows associations of the BCAA factor score with change in the metabolic biomarkers in boys. A higher BCAA score corresponded with decreased C-peptide, CP-IR, total cholesterol, and LDL cholesterol during follow-up, with consistent estimates across the two multivariable models. For example, in Model 1, each 1 unit increase in the BCAA score corresponded with a 0.17 (95\% confidence interval $(\mathrm{Cl}):-0.08,0.42) \mathrm{ng} / \mathrm{mL}$ decrease in $\mathrm{C}$ peptide, and 0.03 (95\% Cl: $-0.02,0.09)$ unit decrease in CP-IR after adjusting for baseline age, pubertal status, and biomarker level; and age at follow-up. Further adjustment for baseline BMI z-score in Model 2 strengthened these estimates (C-peptide: $-0.28(95 \%$ $\mathrm{Cl}:-0.50,-0.06)$; CP-IR: $-0.05(95 \% \mathrm{Cl}:-0.10,-0.01)$ per 1 unit of the BCAA score). Similarly, a higher BCAA score was related to a decrease in total and LDL cholesterol (total cholesterol: -5.19 (95\% Cl: $-9.60,-0.78) \mathrm{mg} / \mathrm{dL}$; LDL: -4.08 (95\% Cl: $-7.56,-0.60)$ $\mathrm{mg} / \mathrm{dL}$ per 1 unit of the BCAA score) in Model 1, with similar associations in Model 2.

Supplemental Table S1 shows associations of the BCAA pattern with change in the metabolic biomarkers, separately for prepubertal and pubertal girls. While we did not detect any statistically significant associations in pre-pubertal girls, the BCAA pattern was related to an increase in C-peptide and leptin in pubertal girls. Each 1 unit increase in the BCAA score corresponded with 0.30 (95\% Cl: $0.02,0.58) \mathrm{ng} / \mathrm{mL}$ greater increase in Cpeptide, and 6.07 (95\% Cl: 1.68, 10.45) ng/mL greater increase in leptin (Model 1). Further adjustment for baseline BMI $z$-score (Model 2) did not change the direction, magnitude, or precision of these estimates.

Supplemental Table S2 shows associations of individual metabolites within the BCAA pattern with biomarkers of glycemia, and total and LDL cholesterol in boys, while also accounting for covariates in Model 2. All metabolites were associated with a decrease in C-peptide, with the strongest associations observed for isoleucine $(-0.12$ (95\% Cl: $-0.23,-0.02) \mathrm{ng} / \mathrm{mL}$ per 1 
metabolite $z$-score), tyrosine $(-0.15(95 \% \mathrm{Cl}:-0.28,-0.02) \mathrm{mg} / \mathrm{mL}$ units per 1 metabolite $z$-score), and phenylalanine $(-0.11(95 \% \mathrm{Cl}$ : $-0.21,-0.02) \mathrm{ng} / \mathrm{mL}$ units per 1 metabolite $z$-score). We noted similar trends for CP-IR (Table 4). With respect to total and LDL cholesterol, the metabolites were inversely related to both lipids (with the exception of a non-significant positive association between valeryl-carnitine and total cholesterol), with the strongest associations observed for leucine, valine, and tryptophan (Table 4).

A summary of key findings from Model 2 of Supplemental Tables S1 and S2 are displayed in Fig. 1.

Table 3 shows relationships between individual metabolites and the metabolic biomarkers in girls, within strata of pubertal status. In pre-pubertal females, the only association we detected was a small positive relationship between valeryl-carnitine and change in C-peptide $(0.03$ (95\% Cl: 0.00, 0.06) per 1 metabolite $z$-score). On the other hand, in pubertal girls, leucine and phenylalanine were each related to an increase in C-peptide during follow-up (leucine:

Table 2. Associations of the BCAA factor score with change in metabolic biomarkers between baseline (8-14 years) and follow-up (13-19 years) in 85 ELEMENT boys

\begin{tabular}{lll}
\hline & \multicolumn{2}{l}{$\begin{array}{l}\beta(95 \% \mathrm{Cl}) \text { for change in each biomarker per 1 unit } \\
\text { increment in the BCAA factor score }\end{array}$} \\
\cline { 2 - 3 } & Model 1 & Model 2 \\
\hline C-peptide $(\mathrm{ng} / \mathrm{mL})$ & $-0.17(-0.42,0.08)$ & $-\mathbf{0 . 2 8}(-\mathbf{0 . 5 0},-\mathbf{0 . 0 6})$ \\
Glucose $(\mathrm{mg} / \mathrm{dL})$ & $0.46(-1.06,1.98)$ & $0.19(-1.43,1.81)$ \\
CP-IR & $-0.03(-0.09,0.02)$ & $-\mathbf{0 . 0 5}(-\mathbf{0 . 1 0},-\mathbf{0 . 0 1})$ \\
Leptin $(\mathrm{ng} / \mathrm{mL})$ & $-2.57(-5.41,0.28)$ & $-2.67(-5.41,0.08)$ \\
Total cholesterol $(\mathrm{mg} / \mathrm{dL})$ & $-\mathbf{5 . 1 9}(-\mathbf{9 . 6 0},-\mathbf{0 . 7 8})$ & $-\mathbf{5 . 3 5}(-\mathbf{1 0 . 0 2},-\mathbf{0 . 6 7})$ \\
HDL $(\mathrm{mg} / \mathrm{dL})$ & $-0.27(-1.80,1.25)$ & $-0.04(-1.68,1.60)$ \\
LDL $(\mathrm{mg} / \mathrm{dL})$ & $-\mathbf{4 . 0 8}(-\mathbf{7 . 5 6},-\mathbf{0 . 6 0})$ & $-\mathbf{3 . 7 9}(-\mathbf{7 . 4 8},-\mathbf{0 . 0 9})$ \\
Triglycerides $(\mathrm{mg} / \mathrm{dL})$ & $-4.97(-14.09,4.15)$ & $-6.87(-16.43,2.70)$ \\
SBP $(\mathrm{mmHg})$ & $1.41(-0.79,3.61)$ & $-0.03(-2.27,2.21)$ \\
DBP $(\mathrm{mmHg})$ & $0.85(-0.82,2.52)$ & $0.21(-1.56,1.99)$ \\
MetRisk $z$-score & $-0.05(-0.18,0.09)$ & $-0.08(-0.21,0.05)$ \\
\hline
\end{tabular}

a Model 1: Adjusted for age, pubertal status, and the biomarker of interest at baseline, and age at follow-up. Bolded values indicate statistical significance at $a<0.05$. Model 2: Model $1+$ baseline BMI $z$-score

${ }^{b}$ Calculated as the average of five internally standardized sex-specific $z$ scores for inverted HDL, waist circumference, HOMA-IR, triglycerides, and SBP
0.15 (95\% Cl: $0.01,0.29) \mathrm{ng} / \mathrm{mL}$ greater increase per 1 metabolite $z$ score; phenylalanine: $0.10(95 \% \mathrm{Cl}: 0.01,0.20)$ greater increase per 1 metabolite $z$-score). Additionally, leucine, isoleucine, tyrosine, alanine, and propionyl-carnitine were each related to increased leptin during follow-up (Table 3). None of the relationships differed by baseline BMI $z$-score (all $P$-interactions $>0.20$ ).

Table 4 shows results of the LASSO regression. Here, we identified significant predictors of C-peptide and CP-IR as indicators of glycemia (we did not include fasting glucose given that null results in the hypothesis-driven portion of the analysis), as well as leptin, and total and LDL cholesterol. In boys, LASSO regression identified asparagine as a predictor of decreased Cpeptide $(\beta=-0.033 \mathrm{ng} / \mathrm{mL}$ per 1 metabolite $z$-score) and CP-IR ( $\beta$ $=-0.012 \mathrm{mg} / \mathrm{dL}$ per 1 metabolite $z$-score), after accounting for all other metabolites plus covariates in Model 2. Additionally, acetylcarnitine was a positive predictor of change in total cholesterol, whereas 4-hydroxyproline, ornithine, and a-aminoisobutyric acid were each negative predictors of total cholesterol (Table 4). In girls, the only significant relationship was an inverse association between histidine and change in total cholesterol $(\beta=-0.033$ $\mathrm{mg} / \mathrm{dL}$ per 1 metabolite $z$-score). The LASSO model did not identify any metabolites predictive of change in LDL or leptin in either sex, beyond what was accounted for by the covariates in Model 2.

\section{DISCUSSION}

In this prospective study of 179 children aged 8-14 years at baseline, we observed sex-specific and pubertal-stage-specific associations of a BCAA metabolite pattern with conventional biomarkers of metabolic risk. In boys, the BCAA pattern was associated with decreased C-peptide and CP-IR, as well as total cholesterol and LDL cholesterol. When we disaggregated this pattern into its component metabolites, isoleucine, tyrosine, and phenylalanine exhibited the strongest associations with C-peptide and CP-IR; and leucine, valine, and tryptophan were key determinants of total cholesterol and LDL cholesterol. In girls, we found that a higher score for the BCAA pattern at baseline corresponded with increased C-peptide and leptin among those who were classified as pubertal. The association with C-peptide was driven by leucine and phenylalanine; and the association with leptin was driven by leucine, isoleucine, tyrosine, alanine, and propionyl-carnitine. Use of a data-driven technique (LASSO regression) to identify metabolites most strongly predictive of conventional metabolic biomarkers revealed inverse associations

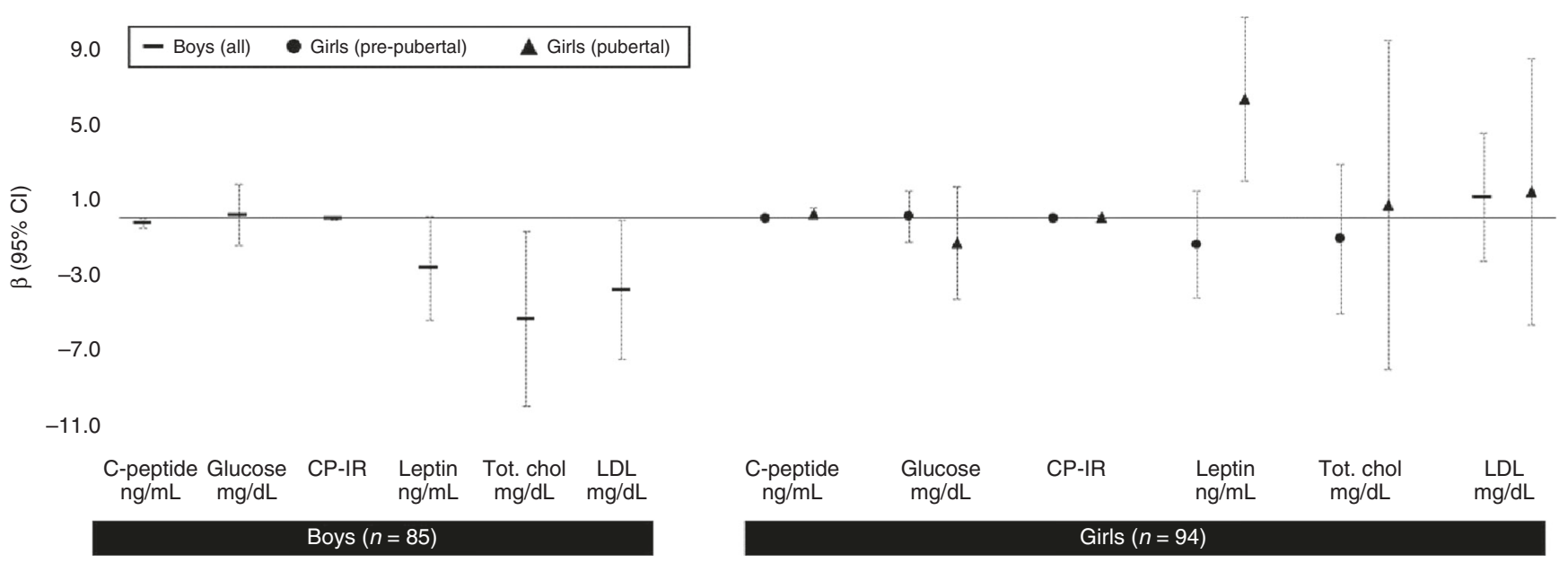

Fig. 1 Associations of the BCAA factor score with change in select metabolic biomarkers between baseline (8-14 years) and follow-up (13-19 years) in 85 boys and 94 girls in ELEMENT. Estimates are adjusted for age, pubertal status, BMl $z$-score, and the biomarker of interest at baseline, and age at follow-up. BCAA branched-chain amino acid, CP-IR C-peptide-based insulin resistance, Tot. chol: total cholesterol, LDL low-density lipoprotein cholesterol 
Table 3. Associations of individual metabolites within the BCAA pattern with change in biomarkers of glycemia in 94 ELEMENT girls, stratified by pubertal status

\begin{tabular}{|c|c|c|c|c|c|c|}
\hline & \multirow[t]{3}{*}{ Mean $\pm S D^{a}$} & \multirow[t]{3}{*}{ Factor loading } & \multicolumn{4}{|c|}{$\beta(95 \% \mathrm{Cl})^{\mathrm{b}}$ for $\Delta$ in each biomarker per $1 z$-score increment in each metabolite } \\
\hline & & & \multicolumn{2}{|l|}{ Pre-pubertal $(n=69)$} & \multicolumn{2}{|l|}{ Pubertal $(n=25)$} \\
\hline & & & C-peptide (ng/mL) & Leptin $(\mathrm{ng} / \mathrm{mL})$ & C-peptide (ng/mL) & Leptin $(\mathrm{ng} / \mathrm{mL})$ \\
\hline Isoleucine & $0.38 \pm 2.04$ & 0.20 & $0.02(-0.07,0.12)$ & $-0.80(-2.32,0.72)$ & $0.11(-0.03,0.26)$ & $3.09(0.67,5.51)$ \\
\hline Valine & $0.20 \pm 1.94$ & 0.19 & $0.03(-0.07,0.13)$ & $-0.54(-2.12,1.04)$ & $0.13(-0.05,0.30)$ & $2.70(-0.38,5.78)$ \\
\hline Tyrosine & $0.34 \pm 1.74$ & 0.18 & $0.04(-0.09,0.16)$ & $-1.16(-3.07,0.75)$ & $0.14(-0.03,0.31)$ & $3.97(1.07,6.87)$ \\
\hline Tryptophan & $0.14 \pm 1.53$ & 0.15 & $-0.03(-0.16,0.11)$ & $-0.03(-2.14,2.08)$ & $0.05(-0.14,0.24)$ & $1.79(-1.49,5.07)$ \\
\hline Alanine & $0.36 \pm 1.77$ & 0.14 & $0.03(-0.09,0.15)$ & $-0.45(-2.31,1.40)$ & $0.05(-0.10,0.20)$ & $2.71(0.20,5.23)$ \\
\hline Propionyl-carnitine & $0.23 \pm 1.72$ & 0.08 & $0.05(-0.07,0.17)$ & $-0.63(-2.49,1.23)$ & $0.01(-0.15,0.18)$ & $2.75(0.04,5.46)$ \\
\hline Valeryl-carnitine & $1.10 \pm 7.41$ & 0.01 & $0.03(0.00,0.06)$ & $0.07(-0.30,0.44)$ & $0.00(-0.13,0.14)$ & $0.83(-1.57,3.24)$ \\
\hline
\end{tabular}

Bolded values indicate statistical significance at $\alpha<0.05$.

${ }^{a}$ Concentrations of each metabolite was coverted to a $z$-score centered at the median and scaled by 1 mean absolute deviation (MAD)

${ }^{b}$ Adjusted for age, pubertal status, BMI z-score and the biomarker of interest at baseline, and age at follow-up

Table 4. Metabolites predictive of change $(\Delta)$ in select metabolic biomarkers in boys using Least Absolute Shrinkage and Selection Operator (LASSO) regression

\begin{tabular}{|c|c|c|c|}
\hline \multicolumn{2}{|l|}{ Boys $(n=85)$} & \multicolumn{2}{|l|}{ Girls $(n=94)$} \\
\hline Metabolite & $\beta^{\mathrm{a}}$ & Metabolite & $\beta^{\mathrm{a}}$ \\
\hline \multicolumn{4}{|l|}{$\Delta$ C-peptide $(\mathrm{ng} / \mathrm{mL})$} \\
\hline Asparagine & -0.033 & - & - \\
\hline \multicolumn{4}{|l|}{$\Delta \mathrm{CP}-\mathrm{IR}$} \\
\hline Asparagine & -0.012 & - & - \\
\hline \multicolumn{4}{|l|}{$\Delta$ Total cholesterol (mg/dL) } \\
\hline Acetyl-carnitine & 2.098 & Histidine & -0.033 \\
\hline 4-Hydroxyproline & -0.050 & - & - \\
\hline Ornithine & -0.353 & - & - \\
\hline$\alpha$-Aminoisobutyric acid & -0.793 & - & - \\
\hline \multicolumn{4}{|l|}{$\Delta \mathrm{LDL}(\mathrm{mg} / \mathrm{dL})$} \\
\hline- & - & - & - \\
\hline \multicolumn{4}{|l|}{$\Delta$ Leptin (ng/mL) } \\
\hline- & - & - & - \\
\hline $\begin{array}{l}\text { aEstimates came from a } \\
\text { baseline age, pubertal statu } \\
\text { follow-up }\end{array}$ & $\begin{array}{l}\text { egres } \\
\text { score }\end{array}$ & $\begin{array}{l}\text { hodel that a } \\
\text { iomarker lev }\end{array}$ & $\begin{array}{l}\text { hted for } \\
\text { d age at }\end{array}$ \\
\hline
\end{tabular}

of asparagine with C-peptide and CP-IR. LASSO regression also detected positive relations of acetyl-carnitine, and inverse associations of 4-hydroxyproline, ornithine, and aaminoisobutyric acid with total cholesterol during follow-up in boys. In girls, histidine was predictive of decreased total cholesterol.

\section{Hypothesis-driven approach}

The inverse relationship of the BCAA pattern with change in Cpeptide and CP-IR was unexpected, as it is the opposite of what has been found in adults. ${ }^{2}$ However, our findings align with results of two cross-sectional investigations of adolescents in Pittsburgh. In the first study, Michaliszyn et al. ${ }^{4}$ reported that several compounds in the BCAA pattern, including leucine, isoleucine, valine, phenylalanine, and propionyl-carnitine, were associated with better insulin sensitivity among 139 adolescents $\sim 13$ years of age. ${ }^{4}$ In the same population, Mihalik et al. found evidence that these compounds were correlated with enhanced fatty acid oxidation, ${ }^{3}$ suggesting that the discrepancy in findings between adults and adolescents may be due to an adaptive increase in mitochondrial function (and accordingly, an improvement in glycemia) during early life that wanes with age and continued metabolic dysregulation. ${ }^{13,24}$ Another explanation for the conflicting literature is that circulating BCAA are a biomarker of impaired insulin action, rather than a direct determinant of insulin resistance. In fact, rodent models have documented beneficial effects of BCAA intake on glycemia that are hypothesized to operate via their nutrient-signaling properties. For instance, leucine activates the mammalian target of rapamycin (mTOR), a nutrient sensor involved in energy balance, food intake, and glycemic regulation, ${ }^{25}$ that has been shown to improve glucose tolerance in mice. ${ }^{26}$

When we examined associations of individual metabolites in relation to the glycemia biomarkers, we detected the strongest associations for isoleucine, tyrosine, and phenylalanine. The inverse relationship of isoleucine with insulin secretion (C-peptide) and resistance (CP-IR) aligns with findings from in vitro and in vivo studies documenting a beneficial effect of isoleucine on skeletal muscle glucose uptake ${ }^{27}$ and whole body glucose oxidation. ${ }^{28}$ Because both of these processes reduce circulating glucose levels without the involvement of insulin, higher circulating isoleucine could theoretically reduce insulin secretion and protect against insulin resistance. The inverse relationship of tyrosine and its precursor phenyalanine with C-peptide and CP-IR may be related the actions of tyrosine kinase, an enzyme composed of tyrosine and a phosphate group that improves efficacy of insulin signaling. ${ }^{29}$ Ultimately, the above-mentioned mechanisms would function to reduce the amount of insulin required to elicit the necessary physiological response.

The BCAA pattern-in particular, leucine, valine, and tryptophan -was associated with lower total and LDL cholesterol. While we were not able to locate any published studies that specifically focused on BCAAs and change in lipid profile, there are a few potential explanations for our findings. The first is that the inverse association between BCAAs and lipids is secondary to the relationship between BCAAs and glycemia, which is plausible given that dysglycemia (i.e., insulin resistance) explains a significant portion of variability in circulating lipid levels during adolescence. ${ }^{30}$ Another explanation is that our finding is actually reflective of differences in tempo of sexual maturation. This notion is worth considering given that increased growth hormone 
secretion and protein turnover during puberty may affect BCAA uptake or release from muscle, and can also lead to a decrease in circulating lipid levels that occurs during the age range of our study participants. ${ }^{31,32}$ Thus, although we accounted for pubertal status in the multivariable models, we cannot rule out the possibility of residual confounding by progression sexual maturation.

In pubertal girls, we found a positive association between the BCAA pattern with C-peptide and leptin during follow-up. Leucine and phenylalanine were key determinants of change in C-peptide; and leucine, isoleucine, tyrosine, alanine, and propionyl-carnitine were most strongly associated with change in leptin. Among prepubertal females, we did not find any associations between the BCAA pattern and individual metabolites.

The positive relationship between baseline leucine and phenylalanine, as well as non-significant but positive associations with several other compounds within the BCAA pattern (e.g., isoleucine, valine, tyrosine), with C-peptide in pubertal girls is opposite of what we found in boys, but corroborates published studies in adults, and two small studies in similarly aged youth., The relationship between BCAA and insulin resistance is complex, with some mechanistic studies showing beneficial effects of BCAAs on glycemia, ${ }^{33}$ while others indicate that BCAAs are detrimental. ${ }^{1}$ It is possible that the physiological effects of BCAAs are context-dependent. For example, animal models indicate that the BCAA biochemical pathway is influenced by the hormonal milieu, ${ }^{34}$ which might explain the discrepancy of associations between males and females in this population.

Our finding that the BCAA pattern was associated with increased leptin during follow-up in pubertal girls, even after accounting for BMI $z$-score at baseline, aligns with the fact that BCAAs (leucine, in particular) can stimulate leptin secretion. ${ }^{35}$ The consistent direction of associations of the BCAA pattern with Cpeptide and leptin with respect to this metabolite pattern makes sense in light of the fact that insulin and leptin both act centrally to regulate food intake and glucose homeostasis. ${ }^{36}$

Data-driven approach

To identify novel compounds involved in metabolic risk, we applied LASSO regression to home in on metabolites that are the strongest predictors of change in conventional metabolic biomarkers. We specifically focused on C-peptide, CP-IR, total cholesterol and LDL cholesterol, and leptin based on results of the hypothesis-driven analysis.

In boys, asparagine was the strongest determinant of decreasing C-peptide and CP-IR. This association could transpire from the impact of its derivative, aspartic acid, on secretion of growth hormone, ${ }^{37}$ which is involved in glycemic homeostasis via regulation of glucose oxidation and muscle uptake of glucose. We also identified several metabolites predictive of change in total cholesterol in boys. Acetyl-carnitine, a short-chain acylcarnitine derived from acetyl-CoA involved in lipid oxidation, ${ }^{38}$ was predictive of increasing cholesterol. On the other hand, 4hydroxyproline, a product of collagen degradation that has been implicated in the pathogenesis of atherosclerosis in animal models; ${ }^{39}$ ornithine, an amino acid involved in the urea cycle that modulates lipid metabolism; ${ }^{40}$ and a-aminoisobutyric acid, an isomer of $\beta$-aminoisobutyric acid that is inversely correlated with cardiometabolic risk factors in vitro and in vivo, ${ }^{41}$ were each determinants of a decrease in total cholesterol during follow-up. The LASSO models did not yield any metabolites predictive of change in LDL or leptin in boys, beyond what was already accounted for by the covariates.

In girls, we only identified one statistically significant relationship between metabolites and change in the metabolic biomarkers: histidine predicted a small decrease in total cholesterol. This finding could be explained by histidine's ability to promote lipid peroxidation, ${ }^{42}$ which generally decreases circulating lipid levels.
If BCAAs were the strongest predictors of worsening metabolic health, then the data-driven analysis should have selected those metabolites, which was not the case in this study. The lack of concordance between results of the hypothesis-driven and datadriven analyses can be explained, in part, by the fact that LASSO conditions on (i.e., adjusts for) all other metabolites when estimating $\beta$ for the relationship between each compound and change in the conventional biomarkers, whereas $\beta$ values for the BCAA metabolites represent marginal associations that do not jointly consider other metabolites. Additionally, associations of BCAA metabolites that are on related biochemical pathways (and therefore, are likely correlated with each other) with the outcomes may be attenuated in the LASSO model as it accounts for all other metabolites in consideration.

\section{Strengths and limitations}

Limitations of this study include assessment of plasma metabolites at a single point in time, which precludes our ability to infer on upregulation vs. downregulation of specific pathways and mechanisms; relatively small sample size; potential for falsepositive associations; variation in the tempo of sexual maturation during follow-up; and relatively small sample size, especially when exploring associations within sex-specific strata of pubertal status. Additionally, we acknowledge the potential lack of generalizability of findings to non-Hispanic populations, especially given the role of genetic predisposition of metabolic risk in this ethnic subgroup. ${ }^{43}$ However, the extent to which genetic predisposition manifests as differences in circulating metabolites associated with metabolic risk remains yet to be elucidated.

Strengths of this investigation include the two-pronged approach armed by a hypothesis-driven and data-driven analytical strategy, the former of which reduces the multiple testing, and the latter of which provides an avenue to identify novel biochemical pathways; and our ability to examine the relationship of BCAA and related metabolites with prospective change in multiple metabolic biomarkers in a population afflicted by high rates of obesity and metabolic disease.

\section{CONCLUSIONS}

The BCAA metabolite pattern was associated with prospective change in glycemia and lipid profile in a sex-specific and pubertalstage-specific manner. Contrary to findings in adults, the BCAA pattern was not a good biomarker of metabolic risk in adolescent boys. However, in pubertal girls, this metabolite pattern was associated with increased insulin resistance and leptin during follow-up. Use of LASSO regression identified several additional compounds associated with change in glycemia (asparagine in boys), and total cholesterol (acetyl-carnitine, 4-hydroxyproline, ornithine, and a-aminoisobutyric acid in boys; histidine in girls) that deserve further investigation. Given that many metabolic risk factors considered in this analysis, particularly lipid profile ${ }^{44,45}$ and glycemia, ${ }^{45}$ track from late childhood/early adolescence into adulthood, our findings add to the current literature and represent a step forward in understanding of biomarkers and etiology of metabolic risk during early life. Future studies are warranted to confirm our findings in other populations of adolescents undergoing the pubertal transition, and to explore these associations beyond puberty.

\section{ACKNOWLEDGEMENTS}

This study was funded by the US National Institutes of Health (K24 HD069408, R01 HD 034568, P30 DK092924, R24 DK097153). The targeted metabolomics assays were funded by NIH P30 DK089503 MNORC, NIH 2P30-DK020572 MDTR, NIH UL1TR000433 MICHR, and NIH U24DK097153. The funder was not involved in the design and conduct of the study; collection, management, analysis, or interpretation of the data; and preparation, review, or approval of the manuscript. 


\section{AUTHOR CONTRIBUTIONS}

Substantial contributions to conception and design, acquisition of data, or analysis and interpretation of data: W.P., L.T., P.X.K.S., M.M.T.-R., A.C., K.E.P. Drafting the article or revising it critically for important intellectual content: W.P., L.T., P.X.K.S., M.M.T.-R., A.C., K.E.P. Final approval of the version to be published: W.P., L.T., P.X.K.S., M.M.T.-R., A.C. K.E.P.

\section{ADDITIONAL INFORMATION}

The online version of this article (https://doi.org/10.1038/s41390-018-0195-5) contains supplementary material, which is available to authorized users.

Competing interests: The authors declare no competing interests.

Publisher's note: Springer Nature remains neutral with regard to jurisdictional claims in published maps and institutional affiliations.

\section{REFERENCES}

1. Newgard, C. B. et al. A branched-chain amino acid-related metabolic signature that differentiates obese and lean humans and contributes to insulin resistance. Cell Metab. 9, 311-326 (2009).

2. Wang, T. J. et al. Metabolite profiles and the risk of developing diabetes. Nat. Med. 17, 448-453 (2011).

3. Mihalik, S. J. et al. Metabolomic profiling of fatty acid and amino acid metabolism in youth with obesity and type 2 diabetes: evidence for enhanced mitochondrial oxidation. Diabetes Care 35, 605-611 (2012).

4. Michaliszyn, S. F. et al. Metabolomic profiling of amino acids and beta-cell function relative to insulin sensitivity in youth. J. Clin. Endocrinol. Metab. 97, E2119-E2124 (2012).

5. Perng, W. et al. Metabolomic profiles and childhood obesity. Obesity (Silver Spring). 22, 2570-2578 (2014).

6. McCormack, S. E. et al. Circulating branched-chain amino acid concentrations are associated with obesity and future insulin resistance in children and adolescents. Pediatr. Obes. 8, 52-61 (2013).

7. Lee, A. et al. Prediction of future risk of insulin resistance and metabolic syndrome based on Korean boy's metabolite profiling. Obes. Res. Clin. Pract. 9, 336-345 (2015).

8. Hivert, M. F. et al. Metabolomics in the developmental origins of obesity and its cardiometabolic consequences. J. Dev. Orig. Health Dis. 6, 65-78 (2015).

9. Butte, N. F. et al. Global metabolomic profiling targeting childhood obesity in the Hispanic population. Am. J. Clin. Nutr. 102, 256-267 (2015).

10. $\mathrm{Hu}, \mathrm{H}$. et al. Fetal lead exposure at each stage of pregnancy as a predictor of infant mental development. Environ. Health Perspect. 114, 1730-1735 (2006).

11. Michigan Regional Comprehensive Metabolomics Resource Core (MRC2). Amino Acids: Biofluids, Cell Cultures, Tissues or Feces. http://mrc2.umich.edu/sites/default/ files/Amino\%20Acids\%20-\%20AssayDescription_0.pdf

12. Michigan Regional Comprehensive Metabolomics Resource Core (MRC2). Acylcarnitines. http://mrc2.umich.edu/sites/default/files/Acyl\%20Carnintines\%20-\% 20AssayDescription.pdf

13. Bonser, A. M. \& Garcia-Webb, P. C-peptide measurement: methods and clinical utility. Crit. Rev. Clin. Lab. Sci. 19, 297-352 (1984).

14. Perng, W. et al. Dietary patterns exhibit sex-specific associations with adiposity and metabolic risk in a cross-sectional study in urban Mexican adolescents. J. Nutr. 147, 1977-1985 (2017).

15. Al-Hinai, M. et al. Maternal intake of omega-3 and omega- 6 polyunsaturated fatty acids during mid-pregnancy is inversely associated with linear growth. J. Dev. Orig. Health Dis. 9, 432-441 (2018).

16. Perng, W. et al. Metabolomic determinants of metabolic risk in Mexican adolescents. Obesity (Silver Spring) 25, 1594-1602 (2017).

17. Perng W. et al. Associations of the infancy body mass index peak with anthropometry and cardiometabolic risk in Mexican adolescents. Ann. Hum. Biol. in press (2018).

18. Ekelund, U. et al. Independent associations of physical activity and cardiorespiratory fitness with metabolic risk factors in children: the European youth heart study. Diabetologia 50, 1832-1840 (2007).

19. Viitasalo, A. et al. Validation of metabolic syndrome score by confirmatory factor analysis in children and adults and prediction of cardiometabolic outcomes in adults. Diabetologia 57, 940-949 (2014).
20. Christensen, H. N. Role of amino acid transport and countertransport in nutrition and metabolism. Physiol. Rev. 70, 43-77 (1990).

21. Sattar, N. et al. Elevated alanine aminotransferase predicts new-onset type 2 diabetes independently of classical risk factors, metabolic syndrome, and Creactive protein in the west of Scotland coronary prevention study. Diabetes $\mathbf{5 3}$, 2855-2860 (2004).

22. Tibshirani, R. Regression shrinkage and selection via the Lasso. J. R. Stat. Soc. Ser. B 58, 267-288 (1996).

23. Stone, M. Cross-validatory choice and assessment of statistical predictions. J. $R$. Stat. Soc. Ser. B (Methodol.) 36, 111-147 (1974).

24. Lenaers, E. et al. Adaptations in mitochondrial function parallel, but fail to rescue, the transition to severe hyperglycemia and hyperinsulinemia: a study in Zucker diabetic fatty rats. Obesity (Silver Spring). 18, 1100-1107 (2010).

25. Cota, D. et al. Hypothalamic mTOR signaling regulates food intake. Science $\mathbf{3 1 2}$, 927-930 (2006)

26. Macotela, Y. et al. Dietary leucine-an environmental modifier of insulin resistance acting on multiple levels of metabolism. PLOS ONE 6, e21187 (2011).

27. Doi, M. et al. Isoleucine, a blood glucose-lowering amino acid, increases glucose uptake in rat skeletal muscle in the absence of increases in AMP-activated protein kinase activity. J. Nutr. 135, 2103-2108 (2005).

28. Doi, M., Yamaoka, I., Nakayama, M., Sugahara, K. \& Yoshizawa, F. Hypoglycemic effect of isoleucine involves increased muscle glucose uptake and whole body glucose oxidation and decreased hepatic gluconeogenesis. Am. J. Physiol. Endocrinol. Metab. 292, E1683-E1693 (2007).

29. Cox, M. \& Nelson, D. R. Lehninger: Principles of Biochemistry (W. H. Freeman, New York, Basingstoke, 2008).

30. Steinberger, J., Moorehead, C., Katch, V. \& Rocchini, A. P. Relationship between insulin resistance and abnormal lipid profile in obese adolescents. J. Pediatr. 126, 690-695 (1995).

31. Mascarenhas, L. P., Leite, N., Titski, A. C., Brito, L. M. \& Boguszewski, M. C. Variability of lipid and lipoprotein concentrations during puberty in Brazilian boys. J. Pediatr. Endocrinol. Metab. 28, 125-131 (2015).

32. Eissa, M. A., Mihalopoulos, N. L., Holubkov, R., Dai, S. \& Labarthe, D. R. Changes in fasting lipids during puberty. J. Pediatr. 170, 199-205 (2016).

33. Macotela, Y. et al. Dietary leucine-an environmental modifier of insulin resistance acting on multiple levels of metabolism. PLOS ONE 6, e21187 (2011).

34. Obayashi, M. et al. Estrogen controls branched-chain amino acid catabolism in female rats. J. Nutr. 134, 2628-2633 (2004).

35. Roh, C., Han, J., Tzatsos, A. \& Kandror, K. V. Nutrient-sensing mTOR-mediated pathway regulates leptin production in isolated rat adipocytes. Am. J. Physiol. Endocrinol. Metab. 284, E322-E330 (2003).

36. Marino, J. S., Xu, Y. \& Hill, J. W. Central insulin and leptin-mediated autonomic control of glucose homeostasis. Trends Endocrinol. Metab. 22, 275-285 (2011).

37. Ottaway, J. H. The potentiation of growth hormone by asparagine and tryptophan. Biochem. J. 136, 441-444 (1973).

38. Adams, S. H. et al. Plasma acylcarnitine profiles suggest incomplete long-chain fatty acid beta-oxidation and altered tricarboxylic acid cycle activity in type 2 diabetic African-American women. J. Nutr. 139, 1073-1081 (2009).

39. Abdelhalim, M. A. K., Siiddiqi, N. J., Alhomida, A. S. \& Al-Ayed, M. S. Effects of feeding periods of high cholesterol and saturated fat diet on blood biochemistry and hydroxyproline fractions in rabbits. Bioinform. Biol. Insights 2, 95-100 (2008)

40. Sugino, T., Shirai, T., Kajimoto, Y. \& Kajimoto, O. L-Ornithine supplementation attenuates physical fatigue in healthy volunteers by modulating lipid and amino acid metabolism. Nutr. Res. 28, 738-743 (2008)

41. Roberts, L. D. et al. $\beta$-Aminoisobutyric acid induces browning of white fat and hepatic $\beta$-oxidation and is inversely correlated with cardiometabolic risk factors. Cell Metab. 19, 96-108 (2014).

42. Erickson, M. C. \& Hultin, H. O. Influence of histidine on lipid peroxidation in sarcoplasmic reticulum. Arch. Biochem. Biophys. 292, 427-432 (1992).

43. Goran, M. I. et al. Effects of PNPLA3 on liver fat and metabolic profile in hispanic children and adolescents. Diabetes 59, 3127-3130 (2010).

44. Webber, L. S., Srinivasan, S. R., Wattigney, W. A. \& Berenson, G. S. Tracking of serum lipids and lipoproteins from childhood to adulthood. The Bogalusa Heart Study. Am. J. Epidemiol. 133, 884-899 (1991).

45. Bao, W., Srinivasan, S. R., Wattigney, W. A. \& Berenson, G. S. Persistence of multiple cardiovascular risk clustering related to syndrome $X$ from childhood to young adulthood. The Bogalusa Heart Study. Arch. Intern. Med. 154, 1842-1847 (1994). 